\title{
Interpolación espacial de la abundancia larval de Aedes aegypti para localizar focos de infestación
}

\author{
Larry Niño ${ }^{1}$
}

Forma de citar

Niño L. Interpolación espacial de la abundancia larval de Aedes aegypti para localizar focos de infestación. Rev Panam Salud Publica. 2011;29(6):416-22.

RESUMEN Objetivo. Diseñar e implementar una metodología de vigilancia que localice los focos de infestación de Aedes aegypti con el empleo de larvitrampas y técnicas de interpolación espacial, las cuales permiten estimar la abundancia vectorial de forma continua en el espacio a partir del conteo de individuos colectados en el área de estudio.

Métodos. Se instalaron 228 larvitrampas —a razón de una por manzana-en la zona más densamente poblada de la comuna cinco de Villavicencio (Meta). Con la información regionalizada de la abundancia de larvas se realizaron interpolaciones espaciales con las técnicas polígonos de Voronoi, Kriging ordinario y ponderación de distancias inversas.

Resultados. Se presenta una metodología alternativa para la vigilancia del vector del dengue, basada en el uso de larvitrampas y técnicas de interpolación espacial, con las cuales se obtuvieron mapas de superficie sustentados en observaciones puntuales.

Conclusiones. Los resultados muestran que esta estrategia aventaja a los indices normalmente usados, dado que permite visualizar de manera continua el nivel de infestación vectorial y por ende el riesgo de transmisión de dengue de acuerdo al grado de infestación por A. aegypti. Es de esperar que su adopción contribuya a planificar, optimizar y evaluar con mayor efectividad las actividades de prevención y control.

Palabras clave

Aedes; dengue; vectores de enfermedades; modelos estadísticos; mapa de riesgo; vigilancia epidemiológica, Colombia.

El dengue, actualmente la arbovirosis con mayor dispersión en el mundo, registra entre 50 y 100 millones de casos por año en más de 100 países tropicales y subtropicales endémicos, donde se calcula que hay unos 3500 millones de personas en riesgo de infección (1-7). La dispersión y la persistencia de esta enfermedad se deben principalmente al rápido crecimiento demográfico, los viajes internacionales, la urbanización incon-

\footnotetext{
Oficina de Epidemiología, Secretaría Seccional de Salud de Vichada, Puerto Carreño, Colombia. La correspondencia se debe dirigir a Larry Niño. Correo electrónico: Ininoa@unal.edu.co
}

trolada en los países endémicos, la proliferación de criaderos de vectores y las inadecuadas medidas de control contra el mosquito (1, 6, 8-11).

El principal vector del virus del dengue es Aedes aegypti, una especie antropofílica adaptada a ambientes urbanos, particularmente a viviendas humanas. Se trata de un vector muy eficiente debido a su comportamiento y a su habilidad para la supervivencia, que incluyen la postura de huevos en una gran variedad de recipientes artificiales, la resistencia de sus huevos a la desecación y la capacidad de la hembra de picar en múltiples ocasiones (1-3, 5, 10, 12-14).
Así es que la alta eficiencia del mecanismo de transmisión del virus, junto a las características biológicas del $A$. aegypti, contribuyen a la creciente aparición de brotes de dengue, cuya epidemiología es controlada principalmente por el estado inmune de la población $(1-3,10,11)$. En lugares endémicos como el municipio de Villavicencio, en Colombia, donde se supone que no hay cambios importantes en el estado inmune poblacional, un aumento en la densidad del vector y consecuentemente el contacto vector-humano, podría aumentar la transmisión del virus $(2,3,10)$. La detección de los cambios en la densidad del 
vector es un factor importante en la epidemiología de la enfermedad toda vez que la razón vector/hospedero influye considerablemente en su capacidad transmisora $(3,12)$.

Las técnicas tradicionales de vigilancia de $A$. aegypti usan los índices aédicos de recipientes, de viviendas y de Breteau para determinar el grado de infestación, dispersión y densidad del mosquito en una zona y tiempo determinados. Estos índices se fundamentan en la detección visual de formas inmaduras del vector dentro de recipientes domésticos, técnica considerada poco sensible por la habilidad de las larvas para escapar y su capacidad de permanecer sumergidas por largos períodos de tiempo $(3,5)$. Asimismo, la proporción de viviendas y recipientes infestados con $A$. aegypti no provee información fehaciente sobre la densidad poblacional al registrar como positivo un recipiente o casa sin tener en cuenta la cantidad de formas inmaduras presentes, lo cual quiere decir que para el índice es igual si hay una o cientos de ellas $(3,9,12,14-17)$.

De acuerdo a la detección del vector, sin embargo, las larvitrampas construidas con llantas de automóvil satisfacen las preferencias de ovoposición de $A$. aegypti porque son recipientes oscuros que pueden almacenar suficiente cantidad de agua con poca materia orgánica y proporcionan condiciones de baja evaporación $(9,18,19)$; además, este dispositivo de captura se caracteriza por ser económico y de fácil construcción e implementación.

La interpolación espacial —cuyo empleo para identificar focos de infestación por A. aegypti es el tema del presente trabajo- consiste en usar puntos con valores conocidos, también llamados puntos de control, para estimar una variable en lugares donde se desconoce; también se considera una forma de transformar información puntual en información de superficie, con el objetivo de combinarla con otros datos para facilitar el análisis y la modelación espacial. El resultado de la interpolación espacial depende de un algoritmo computacional o una ecuación matemática en la cual se emplean los datos de los puntos de control —en este caso el número de larvas. Su precisión depende en gran medida del número y la distribución de los valores conocidos: la estimación de la variable estará mayormente influenciada por las larvitrampas más cercanas, así que una estimación efectiva requiere que estos dispositivos de captura se distribuyan uniformemente entre el área de estudio (20-23).

La interpolación puede clasificarse de diferentes formas, según los puntos de control usados durante la estimación, así: es de carácter global si usa todos los puntos y se considera local cuando utiliza sólo una muestra de ellos; se considera exacto si la superficie estimada incluye los puntos de control e inexacto cuando estos valores cambian $\mathrm{y}$, finalmente, es de carácter determinístico cuando no se calculan los errores de las estimaciones y estocástico cuando las estimaciones se acompañan de los errores y sus varianzas $(20,21,23)$.

Los objetivos de este estudio fueron diseñar e implementar una metodología de vigilancia que localice los focos de infestación de $A$. aegypti con el empleo de larvitrampas y técnicas de interpolación espacial, las cuales permiten estimar la abundancia vectorial de forma continua en el espacio a partir del conteo de individuos colectados en el área de estudio.

\section{MATERIALES Y MÉTODOS}

Se realizó un estudio de tipo transversal observacional en el municipio colombiano de Villavicencio, localizado en el departamento del Meta en las coordenadas $04^{\circ} 09^{\prime \prime} 12^{\prime \prime}$ de latitud norte y $73^{\circ} 38^{\prime \prime}$ $06^{\prime \prime}$ de longitud oeste, dentro de la franja que se extiende desde las estribaciones de la cordillera oriental hasta la frontera natural que la separa de las llanuras propiamente dichas, a una altura sobre el nivel del mar de $642 \mathrm{~m}$. El clima, según la clasificación de Thornthwaite, corresponde a superhúmedo medio, caracterizado por presentar factores de humedad entre 201 y 300, precipitaciones anuales entre 2800 $\mathrm{mm}$ y $5270 \mathrm{~mm}$, y temperaturas entre $16,6^{\circ} \mathrm{C}$ y $26,2^{\circ} \mathrm{C}$. La zona a estudiar dentro del municipio corresponde al área más poblada de la comuna cinco, que comprende los barrios Popular, Olímpico, Menegua, Remanso, Villa Ortiz, Camelias, Guadalajara, Cataluña y Danubio.

Se construyeron y adecuaron 228 dispositivos de captura o larvitrampas, constituidas por 1/3 de llantas de automóvil previamente lavadas para descartar eventuales huevos del vector, sujetas con alambre para darles estabilidad y un asidero para colgarlas. Estos dispositivos se colocaron en cada una de las manzanas consideradas en el estudio, dentro de casas localizadas a mitad de manzana, en sitios tranquilos (preferiblemente en el patio trasero de las viviendas), alejadas de sustancias repelentes y protegidas de la lluvia y el sol. La inspección de las larvitrampas no fue superior a siete días después de su colocación, antes del desarrollo de pupas, para así evitar la generación de focos de infestación; las larvas colectadas se fijaron en alcohol al $70 \%$ para su posterior identificación y cuantificación. Se realizaron dos lecturas durante noviembre de 2008, aunque la primera de ellas fue descartada por considerarse de ajuste.

La cartografía base se realizó a partir de un plano digitalizado y regionalizado de la zona de estudio, en el cual se delimitó el área de interés y se enumeraron las manzanas con sus respectivas larvitrampas (en el programa ArcGIS 9.3), donde los polígonos representan las manzanas y los centroides la ubicación aproximada de los dispositivos de captura, dispuestos como se dijo en los patios traseros de viviendas a mitad de manzana.

Los diferentes procedimientos de interpolación aquí expuestos se realizaron usando el mencionado programa ArcGIS 9.3, salvo el análisis exploratorio de la abundancia de larvas y el cálculo y posterior ajuste del semivariograma, llevados a cabo con el programa R Project 2.9. La clasificación de las variables en los distintos niveles de infestación se realizó con el algoritmo Natural Breaks (Jenks) de ArcGIS 9.3, el cual realiza agrupamientos de acuerdo a las características inherentes de los datos, identificando el mejor agrupamiento de valores similares y maximizando las diferencias entre clases.

Los polígonos de Voronoi corresponden a un método que divide un plano en regiones formadas por los lugares más próximos a cada uno de los puntos de control, en este caso larvitrampas. Las líneas que delimitan los polígonos representan puntos del plano equidistante entre dos larvitrampas vecinas, de manera que entre los polígonos obtenidos se encontrarán los puntos del plano cuya distancia al punto de control es menor que la distancia a cualquier otro de estos puntos. Estos polígonos tienen la única propiedad de que cualquier lugar dentro de sí está más estrechamente asociado al atributo de su respectivo punto de control - en este caso la abundancia de larvasque a cualquier otro polígono $(20,23,24)$.

Durante el análisis exploratorio de la abundancia de larvas se examinaron los datos sin tener en cuenta su distribución 
espacial y se identificó la distribución que seguían con el cálculo de parámetros de estadística descriptiva y pruebas de bondad de ajuste (25).

Una vez establecida la normalidad de los datos, se procedió con el cálculo del semivariograma isotrópico experimental y su posterior ajuste a sentimiento a un modelo teórico, lo cual permitió cuantificar el grado y la escala de la variación espacial (21, 22, 25-27). El semivariograma se calculó de acuerdo a la expresión:

$$
\gamma_{(\mathrm{h})}=1 / 2 \mathrm{~N}_{(\mathrm{h})} \Sigma\left[\mathrm{Z}_{(\mathrm{x})}-\mathrm{Z}_{(\mathrm{x}+\mathrm{h})}\right]^{2}
$$

donde $\gamma_{(\mathrm{h})}$ es la semivarianza de la abundancia de larvas recolectadas en las larvitrampas separadas por los intervalos de las distancias $h ; N_{(\mathrm{h})}$ corresponde al número total de pares de larvitrampas separadas por un intervalo de distancia $h ; Z_{(\mathrm{x})}$ es el número de larvas en una localización $x, \mathrm{y} \mathrm{Z}_{(\mathrm{x}+\mathrm{h})}$ es el número de larvas a la distancia del intervalo $h$ desde $x$.

El Kriging es un método geoestadístico de interpolación espacial de carácter global, exacto y estocástico. La idea básica de este método corresponde a la noción de dependencia espacial, según la cual las muestras cercanas tienen mayor similitud entre sí que las más apartadas. La variación espacial puede deberse a tres componentes: la correlación espacial representada por la variación de la variable regionalizada, la direccionalidad o estructura representada por tendencias y el error aleatorio (20-23, 25, 27, 28). Los estimadores del tipo Kriging son variantes del estimador lineal básico $Z^{*}{ }_{(x)}$, definido como:

$$
Z_{(\mathrm{x})}^{*}-\mathrm{m}_{(\mathrm{x})}=\Sigma \omega_{(\mathrm{i})}\left[\mathrm{Z}_{(\mathrm{xi})}-\mathrm{m}_{(\mathrm{xi})}\right]
$$

donde, $\omega_{(\mathrm{i})}$ son los pesos asignados a los datos $Z_{(\mathrm{xi})}$, los cuales se relacionan con la magnitud y proximidad de las abundancias, y cuyos atributos son estimados en el semivariograma; los valores esperados de las variables aleatorias $Z_{(\mathrm{x})}$ y $Z_{(\mathrm{xi})}$ son $m_{(\mathrm{x})}$ y $m_{(\mathrm{xi})}$ respectivamente; el número de datos $n$, considerado en la estimación, varía de un lugar a otro. En la práctica se emplean los datos existentes en las proximidades del punto a estimar, dentro de un entorno definido al inicio.

La ponderación de distancias inversas (IDW, por sus siglas en inglés) es un método local, exacto y determinístico, donde el grado de influencia o peso de los puntos cercanos es expresado por el inverso de la distancia elevado a un exponente. Un exponente de 1,0 significa que la tasa de cambio es constante entre los puntos (interpolación lineal), mientras que un exponente de 2,0 ó más sugiere que la tasa de cambio de los valores es mayor cerca de puntos conocidos y su influencia se mantiene a mayor distancia $(20-23,27)$. Matemáticamente, el interpolador general IDW se define como:

$$
\mathrm{ID}_{\mathrm{p}}=\Sigma \mathrm{Z}_{(\mathrm{x})} \mathrm{d}^{-\mathrm{p}} / \Sigma \mathrm{d}^{-\mathrm{p}}
$$

donde $I D_{\mathrm{p}}$ es el interpolador; $d$ es la distancia de separación de un punto en el plano a una larvitrampa con abundancia de larvas $Z_{(x)}$; y $p$ el exponente de ponderación.

Para estimar la precisión de los interpoladores IDW y Kriging, en cada caso se realizó una validación cruzada que omitió uno de los puntos a la vez y lo calculó con el resto de puntos de control, comparando así los valores medidos con los valores estimados. Con la diferencia de estos últimos valores se realizó el cálculo de la raíz del error cuadrático medio (RMS, por sus siglas en inglés), el cual establece la precisión de la interpolación de acuerdo a su cercanía a cero $(23,27)$.

FIGURA 1. Disposición de larvitrampas (azul) y abundancia de larvas (rojo) en la comuna cinco de Villavicencio, Colombia, 2008

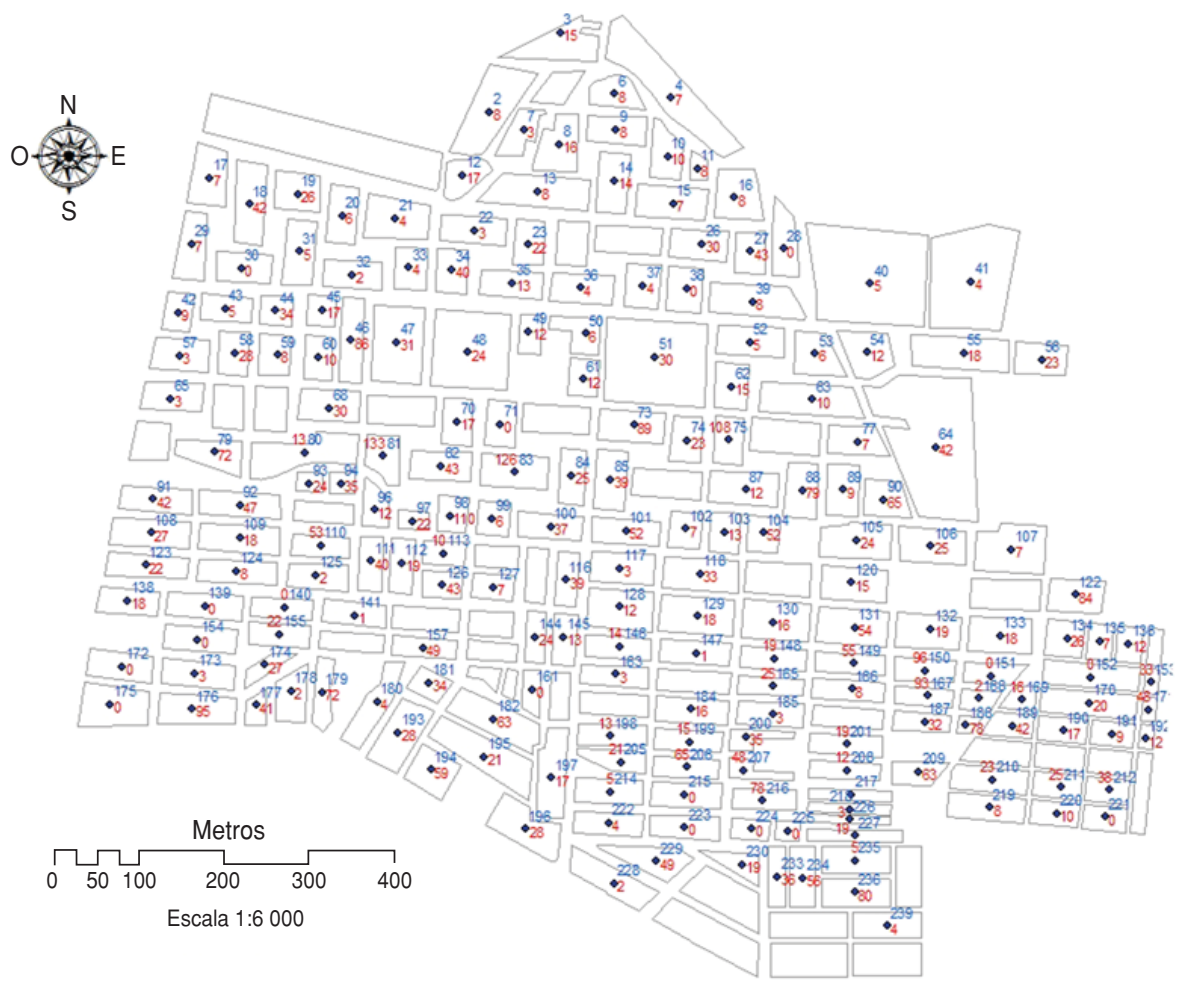

Fuente: elaborada por el autor.

\section{RESULTADOS}

Se colectaron en total 4922 ejemplares de A. aegypti en 185 larvitrampas efectivas. Para facilitar la visualización de la variable en el área de estudio, la abundancia de las larvas recolectadas en cada uno de los dispositivos de captura fue asociada a sus respectivas manzanas (figura 1).

La zona de estudio se dividió merepresentan el área de influencia de la abundancia vectorial estimada con cada una de las larvitrampas instaladas. En el mapa de la figura 2 se puede ver la variable en una clasificación de cinco niveles de infestación que van desde "muy bajo" hasta "muy alto". Los polígonos que representan los mayores niveles de infestación se distribuyen horizontalmente hacia el centro y en la zona suroriental del área de estudio.

El número máximo de larvas en un dispositivo fue de 133 y el mínimo de 2 , con un promedio de 26,6 ejemplares por larvitrampa. La distribución de los datos presenta una asimetría de 1,7, curtosis de 5,9 y desviación estándar (DE) de 25,8. diante polígonos de Voronoi, los cuales 
FIGURA 2. Niveles de infestación aédica en la comuna cinco de Villavicencio, obtenidos mediante interpolación de polígonos de Voronoi, Colombia, 2008

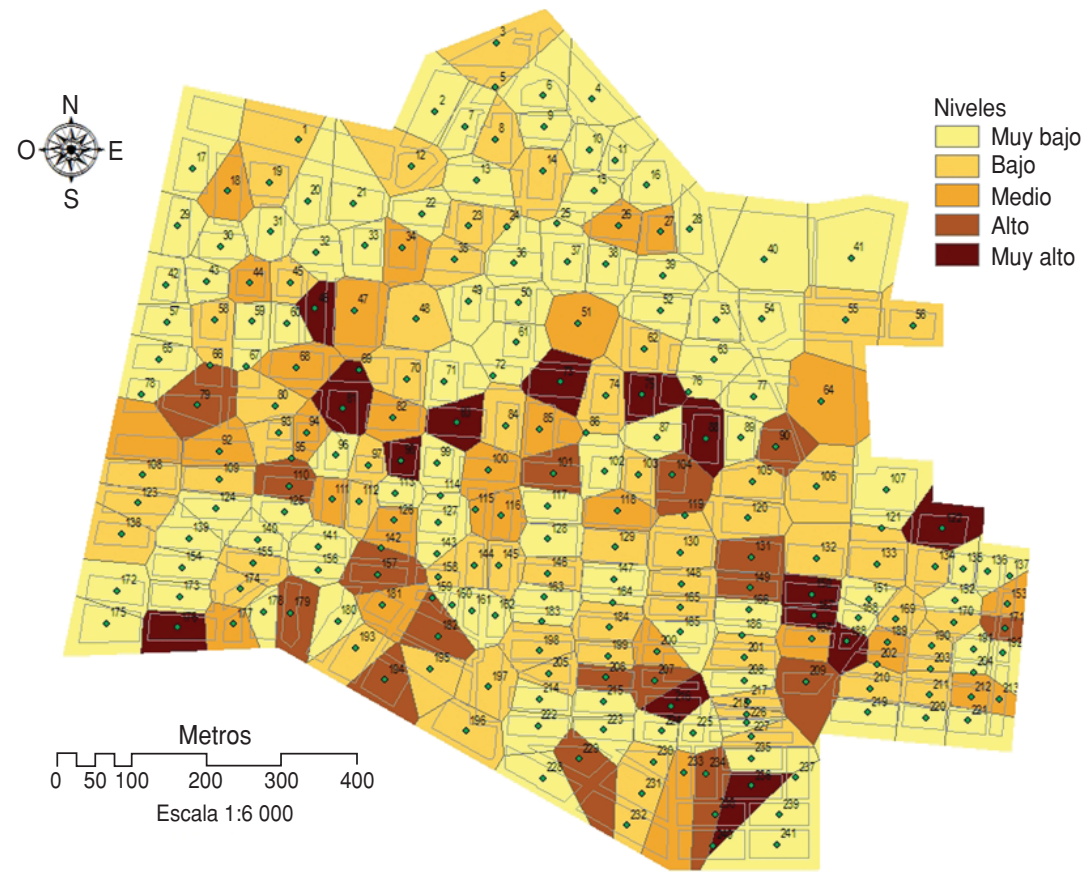

Fuente: elaborada por el autor.
Los anteriores estadísticos descriptivos, el histograma (figura $3 \mathrm{~A}$ ) y el gráfico de probabilidad normal o normal Q-Q Plot (figura 4A) evidencian la presencia de valores extremos que afectan la normalidad de los datos, los cuales se agrupan entre 2 y 40.

Para averiguar si la abundancia larval siguió una distribución normal, se calculó la prueba de Lilliefors (KolmogorovSmirnov), cuyo estadístico resultante fue de 0,17 con una significación de 7,8 e-15. De acuerdo a la significación de la prueba $(P<0,05)$, se rechazó la hipótesis de normalidad de la variable.

FIGURA 3. Abundancia larval antes y después de la transformación logarítmica, comuna cinco de Villavicencio, Colombia, 2008

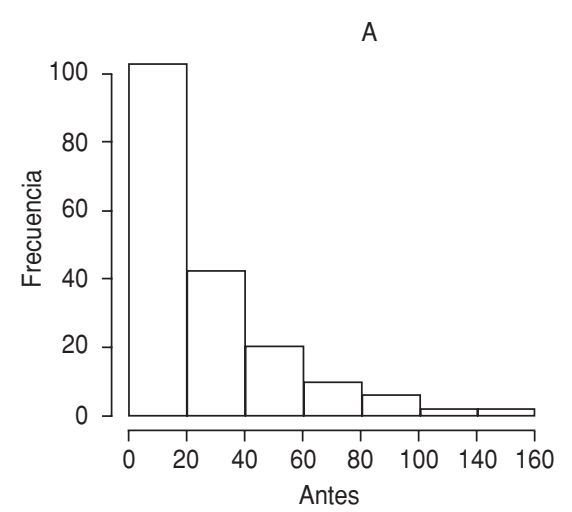

Fuente: elaborada por el autor.
Dado que los datos de la abundancia larval no siguieron una distribución normal, se transformaron con el logaritmo natural para normalizarlos. La distribución de los datos transformados presentó curtosis de 2,3, asimetría de -0,1, histograma de forma acampanada y gráfico de probabilidad normal con forma rectilínea, al menos en su porción central, obteniéndose así indicios de normalidad (figuras 3B y 4B). Para corroborar lo anterior, se calculó de nuevo la prueba de Lilliefors (Kolmogorov-Smirnov), cuyo estadístico resultante fue de 0,05 con una significación de 0,21 , permitiendo acep- tar la hipótesis de normalidad de la variable transformada $(P>0,05)$.

Una vez demostrada la normalidad de la variable, se estableció el grado y la escala de su variación espacial con el cálculo del semivariograma isotrópico clásico por defecto, el cual se ajustó a sentimiento al modelo matemático esférico con el uso del paquete Eyefit de R-Project, hasta una distancia máxima de $430 \mathrm{~m}$ (figura 5). En este modelo, el semivariograma tiende asintóticamente a la semivarianza y se define como:

$$
\gamma_{(\mathrm{h})}=1,5 \mathrm{~h} / \mathrm{a}-0,5(\mathrm{~h} / \mathrm{a})^{3}
$$

donde $\gamma_{(\mathrm{h})}$ es la semivarianza a la distancia $h$, y $a$ el rango del semivariograma.

Los parámetros obtenidos del semivariograma ajustado indican que la abundancia larval se correlaciona espacialmente hasta $80 \mathrm{~m}$ de acuerdo al rango, el cual equivale a la distancia aproximada de una manzana. La meseta y pepita correspondieron a 0,99 y 0,25 respectivamente, indicando que la meseta equivale a aproximadamente el valor de la varianza de los datos $(21,25)$ y que el efecto pepita es despreciable, puesto que la relación pepita/meseta fue menor a 30\% (29).

De acuerdo a los parámetros del semivariograma ajustado, se realizó el mapa de interpolación usando una predicción lineal y asumiendo una media conocida con el método Kriging ordinario, en cuya validación cruzada se obtuvo un RMS de 1,07 (21). La abundancia larval transformada se clasificó en cinco niveles de infestación, de muy bajo a muy alto. Si se consideran como focos a las áreas con niveles de infestación muy altos y altos a la vez, en el área de estudio se observan cuatro focos de infestación, dos en la región suroriental y dos en la porción central. El mayor de los focos tiene una disposición horizontal e involucra al menos 20 manzanas en el centro de la región estudiada (figura 6).

En la interpolación IDW se usó el dos como exponente, al considerarse que la tasa de cambio de la abundancia larval es mayor cerca de los puntos de muestreo y que su influencia se mantiene con la distancia. En el cálculo de interpolación se incluyeron los cinco vecinos más cercanos en un sector de $40 \mathrm{~m}$ a la redonda, y de acuerdo a la validación cruzada se obtuvo un RMS de 1,09.

Con relación a los focos, considerados como áreas con niveles de infestación altos y muy altos, se observa que el mayor 
FIGURA 4. Probabilidad normal de abundancia larval antes y después de la transformación logarítmica, comuna cinco de Villavicencio, Colombia, 2008

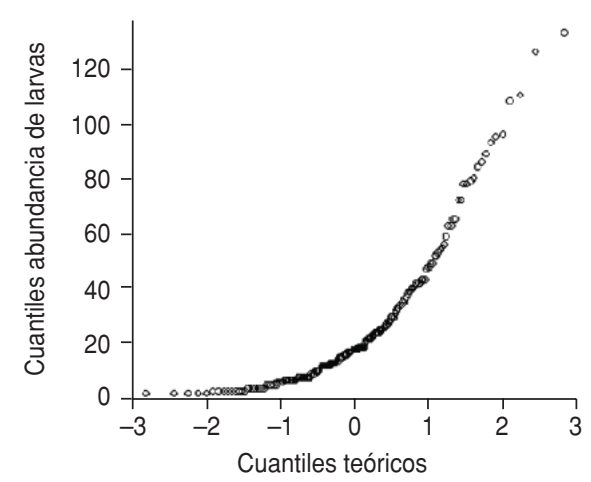

Fuente: elaborada por el autor.

FIGURA 5. Semivariograma isotrópico ajustado del logaritmo natural de la abundancia de larvas en la comuna cinco de Villavicencio, Colombia, 2008

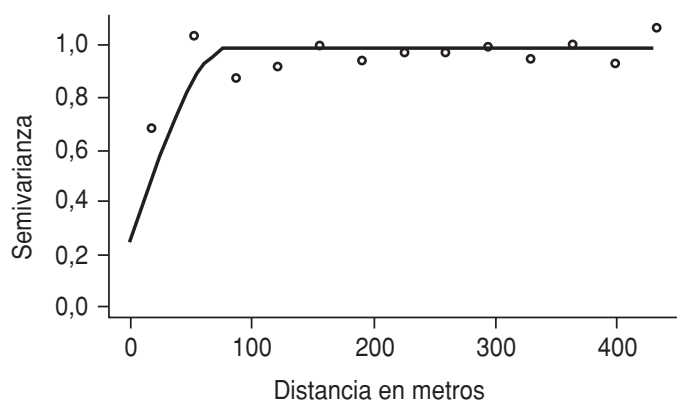

Fuente: elaborada por el autor.

FIGURA 6. Niveles de infestación aédica en la comuna cinco de Villavicencio, obtenidos mediante interpolación Kriging ordinario, Colombia, 2008

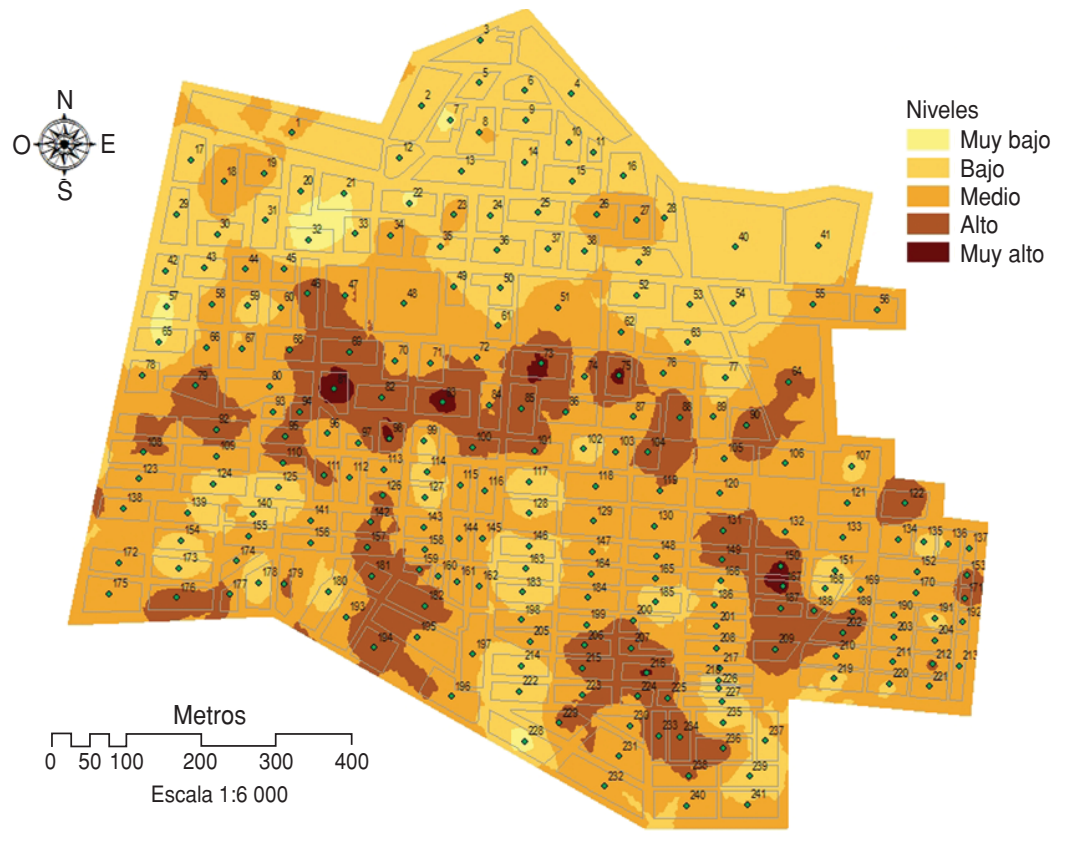

Fuente: elaborada por el autor. se localiza en el centro de la zona estudiada, extendiéndose de forma horizontal e involucrando al menos 20 manzanas de la localidad. Se observan otros siete focos de menor envergadura, cinco de los cuales se distribuyen en el margen meridional del área de estudio y los dos restantes en cada uno de los extremos del mayor de los focos de infestación (figura 7).

\section{DISCUSIÓN}

Los avances en la capacidad de cómputo y las nuevas herramientas de análisis permiten y exigen la búsqueda de metodologías alternativas a los índices aédicos tradicionales, los cuales se implementan ampliamente desde hace ocho décadas sin lograr aún resultados convincentes en la medición del grado de infestación, dispersión y densidad del mosquito en una zona determinada por varias razones, entre ellas i) se basan en la presencia o ausencia de larvas en recipientes domésticos que no siempre presentan condiciones óptimas de visibilidad, ii) vinculan extensas áreas políticoadministrativas con bajos niveles de detalle iii) que no siempre se correlacionan con la transmisión del dengue (1-5, 7-10, $12,14,15,17,19,25,30-34)$.

El uso de larvitrampas permite estimar con mayor precisión la abundancia de $A$. aegypti al proporcionar información fidedigna sobre el número de ejemplares que habitan en distintos puntos del área de estudio. A partir de los datos colectados con larvitrampas se obtienen mapas de interpolación, donde se visualiza de forma continua el nivel de infestación vectorial y consecuentemente el riesgo de infección de dengue según la mayor o menor abundancia del mosquito. Con esta información se puede entonces planificar, mejorar y evaluar actividades de control vectorial con mayor precisión, junto con estrategias de información, educación y comunicación públicas encaminadas a reducir la abundancia de mosquitos y la incidencia de dengue en zonas específicas (3-5, 10, 14, 25, 30-32, 34). Adicionalmente, estos mapas de infestación pueden combinarse fácilmente con información ambiental, demográfica o epidemiológica, y así obtener modelos detallados que tengan la capacidad de monitorear, simular o predecir la transmisión del dengue (4, $5,8,10,12,17,25,30-32$ ).

En las interpolaciones obtenidas, los focos de infestación presentaron distri- 
FIGURA 7. Niveles de infestación aédica en la comuna cinco de Villavicencio, obtenidos mediante interpolación de ponderación de distancias inversas, Colombia, 2008

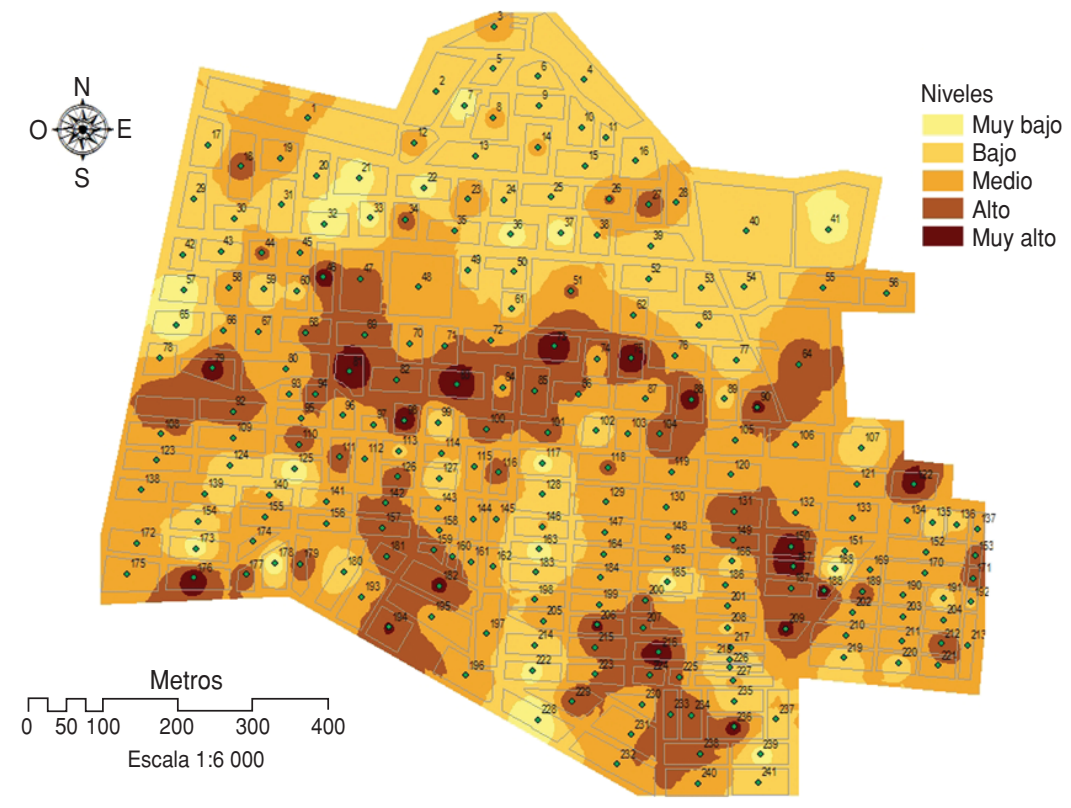

Fuente: elaborada por el autor.

buciones espaciales y tamaños similares, aunque de acuerdo a los RMS, el Kriging ordinario presentó mayor precisión que el IDW.

El ajuste del semivariograma al modelo esférico en la interpolación Kriging fue aceptable, al considerarse el efecto pepita como nulo y la similitud de la varianza de la muestra y la meseta del semivariograma $(25,29)$. La distancia máxima de $80 \mathrm{~m}$ con la cual se correlaciona la variable es aproximadamente la longitud de una manzana, indicando que los focos de infestación inciden a lo sumo en las manzanas circundantes a pesar de la capacidad de $A$. aegypti de dispersarse a distancias superiores, lo cual podría deberse a la disponibilidad de alimento y criaderos dentro de las manzanas $(3,4,6$, $14,17,25,35,36$ ).

Cabe señalar que la metodología aquí expuesta, aun cuando está claro que podría aportar mucha más efectividad a los esfuerzos contra la infestación vectorial que propaga el dengue, no está exenta de limitaciones, en particular las que plantea su focalización específica en las larvas por su facilidad de captura. El punto débil de este enfoque radica en que idealmente la abundancia de hembras adultas estaría relacionada de forma más estrecha con la transmisión del dengue, aparte de que su capacidad de volar confiere continuidad a su distribución espacial, la cual es una característica conveniente a la geoestadística.

\section{Conclusiones}

Se ha diseñado e implementado una metodología de vigilancia entomológica que localiza focos de infestación de $A$. aegypti - mediante el uso de larvitrampas- para generar información regionalizada sobre la abundancia larval y técnicas de interpolación espacial que permiten visualizar de forma continua el nivel de infestación vectorial. Los mapas de superficie generados con esta metodología indican, con mayor detalle que los índices aédicos tradicionales, los lugares específicos donde sería necesario tomar medidas de prevención y control de acuerdo al grado de infestación, permitiendo así una mayor racionalización de tiempo y recursos. Asimismo, el número de larvas constituye una variable que estima la abundancia de adultos de forma mucho más fehaciente que la presencia o ausencia de larvas en recipientes o viviendas usados en los índices aédicos tradicionales. En este sentido, sería recomendable la creación de modelos pormenorizados de transmisión del dengue, combinando mapas de infestación aédica con información regionalizada de carácter epidemiológico, como por ejemplo los casos de dengue notificados, el estado inmunológico de la población y los serotipos virales circulantes; información demográfica, como la densidad de población humana y los estratos socioeconómicos y, finalmente, información ambiental con variables propias de los distintos entornos urbanos. Asimismo, la obtención periódica de mapas de infestación aédica permitiría detectar cambios en la abundancia y distribución de $A$. aegypti debidos a variaciones climáticas o a las medidas preventivas o de control contra el mosquito, lo cual contribuiría a identificar acciones efectivas para la reasignación de esfuerzos y recursos.

Agradecimientos. El autor agradece a la Secretaría Seccional de Salud del Meta y a la Secretaría Local de Salud de Villavicencio por la financiación de este trabajo. Además desea expresar su gratitud a Marina Stella González, Jorge Romero y Omar Ramírez por su gestión administrativa y a Paola Isaacs, Ana María Gómez, Cateryn Romero, Fabriciano Niño y Lucía Gualteros por sus aportes a la redacción del manuscrito.

\section{REFERENCIAS}

1. Kyle J, Harris E. Global spread and persistence of dengue. Annu Rev Microbiol. 2008; 62:71-92.

2. Halstead S. Dengue Virus-Mosquito Interactions. Annu Rev Entomol. 2008;53:273-91.

3. Regis L, Monterior AM, Varial MA, Silveira JC Jr, Furtado AF, Acioli RV, et al. Develop- ing new approaches for detecting and preventing Aedes aegypti population outbreaks: basis for surveillance, alert and control system. Mem Inst Oswaldo Cruz. 2008;103: 50-9.

4. Honório NA, Nogueira RM, Codeço CT, Carvalho MS, Cruz OG, Figuereido MA, et al.
Spatial Evaluation and Modeling of Dengue Seroprevalence and Vector Density in Rio de Janeiro, Brazil. PLoS Negl Trop Dis. 2009; 3:e545.

5. Regis L, Souza W, Furtado A, Fonseca C, Silveira J Jr, Ribeiro P Jr, et al. An entomological surveillance system based on open spatial 
information for participative dengue control. An Acad Bras Cienc. 2009;81:655-62.

6. Chang A, Parrales M, Jimenez J, Sobieszczyk M, Hammer S, Copenhaver D, et al. Combining Google Earth and GIS mapping technologies in a dengue surveillance system for developing countries. Int J Health Geogr. 2009;8:49.

7. Teixeira MG, Nascimento $N$, Barreto $M$, Mota E. Dengue and dengue hemorrhagic fever epidemics in Brazil: what research is needed based on trends, surveillance, and control experiences? Cad Saude Publica. 2005;21:1307-15.

8. Barreto F, Teixeira MG, Nascimento N, Carvalho M, Barreto M. Spread pattern of the first dengue epidemic in the city of Salvador, Brazil. BMC Public Health. 2008;8:51.

9. Marquetti MC, Valdés V, Aguilera L, Navarro A. Vigilancia entomológica de Aedes (S) aegypti y otros culícidos en Ciudad de La Habana, Cuba 1991-1996. Rev Cubana Med Trop. 2000;52:133-7.

10. Eisen L, Lozano S. Use of Mapping and Spatial and Space-Time Modeling Approaches in Operational Control of Aedes aegypti and Dengue. PLoS Negl Trop Dis. 2009;3:e411.

11. Siqueira J Jr, Maciel I, Barcellos C, Souza W, Carvalho M, Nascimento N, et al. Spatial point analysis based on dengue surveys at household level in central Brazil. BMC Public Health. 2008;8:361.

12. Corrêa PRL, França E, Bogutchi TF. Infestação pelo Aedes aegypti e ocorrência da dengue em Belo Horizonte, Minas Gerais. Rev Saude Publica. 2005;39:33-40.

13. Fernández W, Iannacone J, Rodríguez E, Salazar N, Valderrama B, Morales AM. Comportamiento poblacional de larvas de Aedes aegypti para estimar los casos de Dengue en Yurimaguas, Perú, 2000-2004. Rev Peru Med Exp Salud Publica. 2005;22:175-82.

14. Souza R, Carvalho MS. Análise da distribuição espacial de larvas de Aedes aegypti na Ilha do Governador, Rio de Janeiro, Brasil. Cad. Saude Publica. 2000;16:31-42.

15. Bisset JA, Marquetti MC, Portillo R, Rodríguez MM, Suárez S, Leyva M. Factores ecológicos asociados con la presencia de larvas de Aedes aegypti en zonas de alta infestación de Ciudad de La Habana, Cuba. Rev Panam Salud Publica. 2006;19:379-84.

16. Morato V, Teixeira MG, Gomes A, Bergamaschi D, Barreto M. Infestation of Aedes aegypti estimated by oviposition traps in Brazil. Rev Saude Publica. 2005;39:553-8.

17. Donalísio MR, Glasser CM. Vigilância Entomológica e Controle de Vetores do Dengue. Rev Bras Epidemiol. 2002;5:259-72.

18. Honório NA, Lourenço R. Freqüência de larvas e pupas de Aedes aegypti e Aedes albopictus em armadilhas, Brasil. Rev Saude Publica. 2001;35:385-91.

19. Braga IA, Gomes AC, Nelson M, Mello RC, Bergamaschi DP, Pacheco JM. Comparação entre pesquisa larvária e armadilha de oviposição, para detecção de Aedes aegypti. Rev Soc Bras Med Trop. 2000;33:347-53.

20. Albrecht J. Key concepts \& techniques in GIS. London: SAGE Publications Ltd; 2007.

21. Waller L, Gotway C. Applied Spatial Statistics for Public Health Data. New Jersey: Wiley-Interscience; 2004.

22. Pfeiffer D, Robinson T, Stevenson M, Stevens $\mathrm{K}$, Rogers D, Clements A. Spatial analysis in epidemiology. New York: Oxford University Press; 2008.

23. Chang K. Introduction to Geographic Information Systems. 4. ${ }^{\text {a }}$ ed. New York: McGrawHill; 2006.

24. Puga H. Conjuntos de Voronoi, triangulación de Delaunay y generación de caminos. Gaceta ide@s CONCYTEG. 2008;31:3-6.

25. Niño L. Uso de la función semivariograma y estimación Kriging en el análisis espacial de un indicador entomológico de Aedes aegypti (Diptera: Culicidae). Biomedica. 2008;28:578-86.

26. Isaaks E, Srivastava R. An introduction to applied geostatistics. New York: Oxford University Press; 1989.

27. Villatoro M, Henríquez C, Sancho F. Comparación entre los interpoladores IDW y Kriging en la variación espacial de $\mathrm{pH}, \mathrm{Ca}, \mathrm{CICE}$, y $\mathrm{P}$ del suelo. Agronomía Costarricense. 2008;32:95-105.
28. Liebhold AM, Rossi RE, Kemp WP. Geostatistics and geographic information systems in applied insect ecology. Annu Rev Entomol. 1993;38:303-27.

29. Valbuena CA, Martínez LJ, Giraldo R. Variabilidad espacial del suelo y su relación con el rendimiento de mango (Mangifera indica L.). Rev Bras Frutic. 2008;30:1146-51.

30. Barcellos C, Kreutz A, Weber MA, Varnieri MR. Identificação de locais com potencial de transmissão de dengue em Porto Alegre através de técnicas de geoprocessamento. Rev Soc Bras Med Trop. 2005;38:246-50.

31. Lagrotta M, Silva W, Souza-Santos R. Identification of key areas for Aedes aegypti control through geoprocessing in Nova Iguaçu, Rio de Janeiro State, Brazil. Cad Saude Publica. 2008;24:70-80.

32. Ali M, Wagatsuma $Y$, Emch M, Breiman R. Use of a geographic information system for defining spatial risk for dengue transmission in bangladesh: role for aedes albopictus in an urban outbreak. Am J Trop Med Hyg. 2003; 69:634-40.

33. Salas MA, Reyes F. Variación estacional de las poblaciones de Aedes aegypti en Monterrey, México. Salud Publica Mex. 1994;36:385-92.

34. Vargas M. Uso de ovitrampas en los programas de prevención y control del dengue. Rev Col de MQC de Costa Rica. 2002;8:120-4.

35. Fantinatti E, Duque J, Silva A, Navarro-Silva M. Abundância e agregação de ovos de Aedes aegypti L. e Aedes albopictus (Skuse) (Diptera: Culicidae) no Norte e Noroeste do Paraná. Neotrop Entomol. 2007;36:960-5.

36. Chadee D, Doon R, Severson D. Surveillance of dengue fever cases using a novel Aedes aegypti population sampling method in Trinidad, West Indies: the cardinal points approach. Acta Trop. 2007;104:1-7.

Manuscrito recibido el 7 de octubre de 2010. Aceptado para publicación, tras revisión, el 17 de febrero de 2011.
ABSTRACT

\section{Spatial interpolation of Aedes aegypti larvae abundance for locating infestation foci}

Key words
Objective. Design and implement a surveillance method for locating Aedes aegypti infestation foci with the use of larvae traps and spatial interpolation techniques, which facilitate the ongoing estimation of vector abundance in the area by counting the individuals collected in the study area.

Methods. A total of 228 larvae traps were installed - at a rate of one per block-in the most densely populated area of commune five of Villavicencio (Meta). With regionalized information on larvae abundance, spatial interpolations were conducted with the Voronoi polygon, ordinary kriging, and inverse distance weighting techniques.

Results. An alternative method for the surveillance of dengue vectors is presented. This method is based on the use of larvae traps and spatial interpolation techniques to obtain area maps supported by specific observations.

Conclusions. The results show that this strategy is better than the indices normally used, since it facilitates continuous visualization of the level of vector infestation and consequently, the risk of dengue transmission, based on the extent of A. aegypti infestation. Implementation of this strategy is expected to contribute to more effective planning, optimization, and evaluation of prevention and control activities.

Aedes; dengue; disease vectors; models, statistical; risk map; epidemiologic surveillance; Colombia. 